\title{
A performance monitoring method for PID control system
}

\author{
Wei Chen ${ }^{1}$, Peihong Wang ${ }^{1 *}$ and Yihua Dong ${ }^{2,3}$ \\ ${ }^{1}$ School of Energy and Environment, Southeast University, Nanjing, Jiangsu Province, 210096, China \\ ${ }^{2}$ Zhejiang Energy Technology Research Institute Co. Ltd, Hangzhou, Zhejiang Province, 311121, China \\ ${ }^{3}$ Key Laboratory of High Efficiency Energy Conservation and Pollutant Control Technology of Thermal Power Generation Zhejiang \\ Province, Hangzhou, Zhejiang Province, 311121, China
}

\begin{abstract}
The change of plant dynamics results in mismatch between the current model and the model used for design, which may have significant impacts on the performance of control system. A method to monitor the influence of the mismatch is proposed, including the estimation of mismatched model and the evaluation of control performance. The estimation method is based on back-propagation (BP) neural network and provide more accurate and reliable estimation effect. Serious mismatch can be alarmed according to evaluation method presented. Simulation results demonstrate the effectiveness of the proposed method.
\end{abstract}

\section{Introduction}

PID controller is widely used in industrial process control. Due to the change of plant dynamics, the parameters of process model may have a large variation which may result in poor performance of PID control system. For identifying whether the operating status of control system is normal or not, it's essential to monitor the change of control performance.

The performance monitoring consists of two parts, including the estimation of mismatched model and the evaluation of control performance [1]. In the literature of model estimation, the model parameters are estimated by optimization method, in which the goal is to find the best parameter to minimize the difference between the estimated and actual step closed-loop response [2-3]. Similarly, the intelligent optimization algorithm such as genetic algorithm (GA) is also applied to the parameter estimation with the same optimization goal [4-5]. For the optimization method, it is critical to select the initial guess values of each model parameter and the unreasonable values may result in local optimum. The intelligent optimization method may also converge to local optimal solutions, which may lead to the unstable results. Therefore, it is necessary to propose an estimation method which can provide more accurate and reliable estimation effect. For the evaluation of control performance, Wang et al. evaluate the potential consequences of MPM from two parts which include load disturbances rejection performance and loop robustness [6]. The performance of set-point tracking is another critical aspect when evaluating the performance of control system, so it also should be considered.
Starting from such motivations, we propose the method for the estimation of mismatched model and the evaluation of control performance. The estimation method is based on building the relationship between the change of the closed-loop response and that of the model parameter. First, several characteristic variables are extracted from the closed-loop responses. Then, the relationship between the relative variations of the characteristic variables and those of the model parameters is set up by back-propagation (BP) neural network. When the variations of characteristic variables are obtained, the mismatched model parameters can be estimated according to the relationship built. Compared with the optimization methods, the proposed method does not require the initial guess values, and avoids the stochastic and unstable estimation results. Furthermore, the evaluation method is developed to assess the influences of MPM based on the study in [6], and the method is extended from the applicable model from FOPTD to SOPTD model.

\section{Problem formulation}

Suppose we have two control loops in the design and monitoring stage respectively as shown in Figure 1. In this study, the control loop is considered as in the design stage when the controller are updated. In the monitoring stage, MPM may appear then a new model of the actual process $G_{p m}(s)$ should be identified. The suffixes "d" and " $\mathrm{m}$ " indicate the variables in the design and monitoring stage, respectively. The closed-loop response is obtained by introducing a unit step change in the set point.

\footnotetext{
${ }^{*}$ Corresponding author's e-mail: phwang@seu.edu.cn
} 


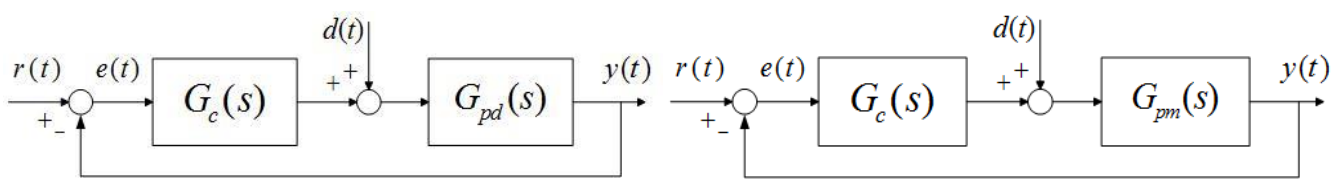

Figure 1. Simplified control loop in the design and monitoring stage.

Consider the process dynamics can be represented as the following two types of models:

(i) First-order-plus-time-delay (FOPTD) model

$$
G_{p}=\frac{K}{T s+1} e^{-\tau s}
$$

(ii) Second-order-plus-time-delay (SOPTD) model

$$
G_{p}=\frac{K}{T^{2} s^{2}+\lambda T s+1} e^{-\tau s}
$$

It is assumed that the process model in the design stage has been obtained by mechanism analysis or system identification in advance. Here, we focus on the identification of the process model in the monitoring stage. When the relationship between the change of the closed-loop response and that of the model parameters is established, the model parameters in the monitoring stage can be estimated by obtaining the closed-loop responses both in the design stage and the monitoring stage. In section 3, the closed-loop responses can be simplified to several characteristic variables. Therefore, when the change of characteristic variables is obtained, the model in the monitoring stage can be estimated according to the relationship between the change of the characteristic variables and that of the model parameters.

With the parameter variations of process model, the performance of control system may be improved or degraded. The control performance includes the set-point tracking performance, load disturbances rejection performance and loop robustness, which are assessed by integral time absolute error $\mathrm{ITAE}_{s}, \mathrm{ITAE}_{l}$ and the maximum sensitivity peak $M_{S}$, respectively. The subscripts "s" and "l" represent the measures of set-point tracking and load disturbances rejection performance, respectively.

ITAE is defined as

$$
\operatorname{ITAE}=\int_{0}^{t} t|e(t)| \mathrm{d} t
$$

where $e(t)=r(t)-y(t)$. For set-point tracking performance, $r(t)$ is chosen as a unit step signal and $d(t)=0$, and $y(t)$ can be obtained by conducting the unit step test. But for load disturbances rejection performance, $y(t)$ need to be obtained by providing the derivation from the process model and controller, $d(t)$ is chosen as a unit step signal and $r(t)=0 . M_{S}$ is denoted as

$$
M_{S}=\max _{0<\omega<\infty}\left|\frac{1}{1+G_{c}(\mathrm{j} \omega) G_{p}(\mathrm{j} \omega)}\right|
$$

For evaluating the change of control performance, the relationship between the model parameter and control performance indices should be established.

ITAE $_{s}$ can be calculated based on the closed-loop response obtained by set-point step test and $M_{S}$ can be calculated with estimated model by (4). The calculation of $\mathrm{ITAE}_{l}$ need to obtain the step closed-loop response of load disturbance, which need the derivation from the process model and controller. When $r(t)=0$ and $d(t)$ is chosen as a unit step signal, the Laplace transforms of $y(t)$ is

$$
Y(s)=\frac{G_{p}(s)}{G_{p}(s) G_{c}(s)+1} D(s)
$$

where $D(s)$ is the Laplace transforms of $d(t)$. Under a closed-loop condition, the process output $y(t)$ for the step test of load disturbance can be obtained by inverse Laplace transform $\mathrm{L}^{-1}[Y(s)]$. PID controller is expressed by $G_{c}(s)=K_{p}\left(1+1 / T_{i} s+T_{d} s\right) . G_{p}(s)$ can be expressed by the form of FOPTD model in (1) or SOPDT model in (2). The process output for FOPTD model has been deduced by Wang et al. [6]. Here, we extend the derivation to SOPDT model.

For SOPTD model, $Y(s)$ is obtained by substituting (2) into (5) with $D(s)=1 / s$

$$
Y(s)=\frac{K T_{i} e^{-\tau s}}{T_{i} T^{2} s^{3}+\lambda T_{i} T s^{2}+T_{i} s+K K_{p}\left(T_{d} T_{i} s^{2}+T_{i} s+1\right) e^{-\tau s}}
$$

To reduce the computational burden, $e^{-\tau s}$ in the fractional expression is approximated by Padé approximation

$e^{-\tau s}=e^{-\tau s / 2} / e^{-\tau s / 2} \approx(1-\tau s / 2) /(1+\tau s / 2) \quad$ and $Y(s)$ is thus approximated as

$$
Y(s)=\tilde{Y}(s) \approx \frac{K T_{i}(\tau s+2) e^{-\tau s}}{a_{4} s^{4}+a_{3} s^{3}+a_{2} s^{2}+a_{1} s+a_{0}}
$$

where

$a_{4}=T_{i} T^{2} \tau, a_{3}=\lambda T_{i} T \tau-K K_{p} T_{d} T_{i} \tau+2 T_{i} T^{2}, a_{2}=2 \lambda T_{i} T+T_{i} \tau+2 K K_{p} T_{d} T_{i}-K K_{p} T_{i} T \tau$, $a_{1}=2 T_{i}+2 K K_{p} T_{i}-K K_{p} \tau, a_{0}=2 K K_{p}$

$y(t)$ can be derived by using the inverse Laplace transform to (7):

$$
y(t)= \begin{cases}0 & , 0 \leq t \leq \tau \\ \sum_{k=1}^{4} \frac{K T_{i}\left(\tau \alpha_{k}+2\right) e^{\alpha_{k}(t-\tau)}}{4 a_{4} \alpha_{k}^{3}+3 a_{3} \alpha_{k}^{2}+2 a_{2} \alpha_{k}+a_{1}}, t>\tau\end{cases}
$$


where $\alpha_{k}$ in (9) is the root of the equation: $a_{4} x^{4}+a_{3} x^{3}+a_{2} x^{2}+a_{1} x+a_{0}=0$.

$\mathrm{ITAE}_{l}$ can be calculated by substituting (9) into (3). The smaller ITAE and $M_{S}$, the better the corresponding performance of control system, and vice versa. According to the above indices calculated based on the mismatched and design model, the influences of the mismatch on the control performance can be assessed by comparing the magnitude and direction of changes in these indices.

\section{Establishment of relationship between characteristic variable and model parameter based on BP neural network}

With the PID controller parameters unchanged, several possibilities exist for the closed-loop response, i.e., according to the shape of the response curve, the response can be divided into three patterns as shown in Figure 2: underdamped, moderate-damped or overdamped.

The closed-loop response consists of a large number of points in time domain, which increases the computing efforts of modelling, so that several characteristic variables are developed to represent the features of the closed-loop response. The following four characteristic variables are developed: $C_{1}=\int_{0}^{t_{d}} t|r(t)-y(t)| d t$, $C_{2}=\int_{0}^{t_{p}} t|r(t)-y(t)| d t, C_{3}=\int_{0}^{t_{s}} t|r(t)-y(t)| d t$, and $C_{4}=y_{t p}$. Here, $t_{d}$ is the time when the closed-loop response reaches $40 \%$ of the final value, and $t_{s}$ is the settling time. For the underdamped or moderate-damped pattern, $y_{t p}$ is the value of the first peak, and $t_{p}$ is the corresponding time. For the overdamped pattern, $y_{t p}$ is defined as the average value of process output when the closed-loop response rises from $60 \%$ to $85 \%$ of the final value, and $t_{p}$ is defined as the time when the closed-loop response reaches $85 \%$ of the final value. The third characteristic variable is the form of ITAE, which can be used to evaluate the control performance.

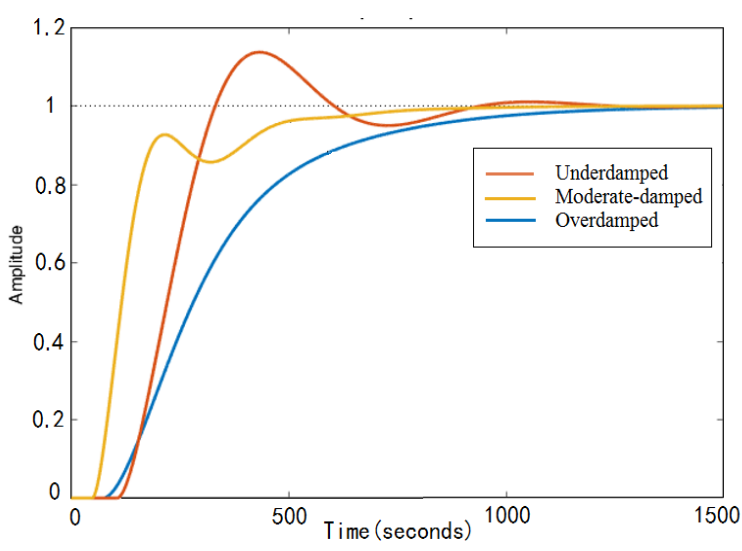

Figure 2. Three patterns of the closed-loop step response

The model parameters and characteristic variables need to be normalized for eliminating the difference among scales. The variation of the $x$ th model parameter is normalized as relative variation:

$$
\eta_{\theta x}=\frac{\theta_{m x}-\theta_{d x}}{\theta_{d x}}
$$

where $\theta_{m x}$ indicates the $x$ th parameter in the mismatched parameter vector $\theta_{m}$. For FOPTD model, $\theta_{m}=\left[K_{m}, T_{m}, \tau_{m}\right]$. For SOPTD model, $\theta_{m}=\left[K_{m}, T_{m}, \tau_{m}, \lambda_{m}\right]$. Similarly, $\theta_{d}$ is the parameter vector in the design stage. The variation of the $x$ th characteristic variable is defined as

$$
\eta_{C x}=\frac{C_{d x}-C_{m x}}{C_{d x}+C_{m x}}
$$

where $C_{m x}$ indicates the $x$ th parameter in the mismatched characteristic variable vector $C_{m}$, and $C_{m}=\left[C_{m 1}, C_{m 2}, C_{m 3}, C_{m 4}\right]$.Similarly, $C_{d}$ is the characteristic variable vector in the design stage.

BP neural network has the advantage of strong nonlinear approximation ability, so we use BP network to set up the mapping relationship between the normalized indices of the characteristic variable and those of model parameters.

The output vector of output layer is $\eta_{\theta}$, and the input vector of input layer is $\eta_{C}$. The training data set consists of the set of $\eta_{\theta}$ and $\eta_{C}$. The set of $\eta_{\theta}$ is obtained from a selected range of model parameters, and the set of $\eta_{C}$ can be calculated from the corresponding closed-loop response. To ensure that the method is applicable in a wide changed range of process dynamics, the range of model parameters selected covers the model parameters at two adjacent operating point.

The neural network toolbox in MATLAB is used to build and train BP neural network. In the neural network toolbox, the training function, adaption learning function and performance function are set as TRAINLM, LEARMGDM and MSE, respectively. Experiments prove that it's feasible to build 3 three-layer BP network 
for 3 closed-loop response patterns, respectively. Each hidden layer has 25 neurons.

\section{Evaluation of control performance}

The control performance of the control system in the design stage is considered as the performance benchmark. With the performance indices calculated in the design and monitoring stage, $\mathrm{ITAE}_{s}, \mathrm{ITAE}_{l}$ and $M_{s}$ can be normalized to $(-1,1)$ by $(11)$, which can be used for depicting the magnitude and direction of the change of the control performance. The positive and negative value of the normalized index indicate improvement and degradation of control performance, respectively. The variation of control performance is larger with a bigger magnitude of corresponding performance index. So the method can reflect the improvement or degradation of control performance clearly.

The process dynamics changes may result in serious mismatch, which leads to the unacceptable degradation on control performance. MPM need alarm when the change of control performance is serious. For performance monitoring, a threshold value is chosen to determine the acceptable variation range of the normalized indices. Here, suppose the threshold values are $3 \%$. When the normalized indices beyond the threshold value, the change of control performance is considered so serious that the mismatch need alarm.
Otherwise, the mismatch is considered acceptable and the alarm is not required.

\section{Simulation Examples}

The proposed method is applied to the process identification of Example 1 and 2 as follow

Example 1:

$$
G_{p d}(s)=\frac{1}{10 s+1} e^{-5 s}, G_{c}(s)=0.7\left(\frac{9 s+1}{9 s}\right)
$$

Example 2:

$$
G_{p d}(s)=\frac{1.2}{61^{2} s^{2}+(2 \times 61) s+1} e^{-81.5 s}, G_{c}(s)=0.42\left(\frac{114.25 s+1}{114.25 s}\right)
$$

Example 1 is a FOPTD model proposed by Wang et al. [6]. Example 2 is a SOPTD model used for approximation of the higher order process in the dashed frame in Figure 3. Figure 3 shows a cascade control system of superheated steam temperature from a boilerturbine unit, and $G_{c 1}=(8 s+0.04) / \mathrm{s}$, $G_{1}(s)=1.657 /(20 s+1)^{2}$ and

$$
G_{2}(s)=1.202 /(27.1 s+1)^{7}[7] \text {. }
$$

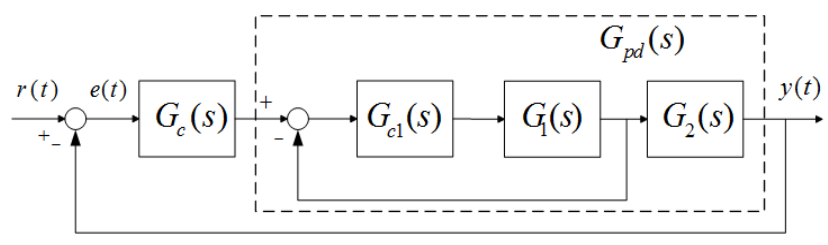

Figure 3. Structure of PID cascade control system

The characteristic variables of closed-loop responses and the indices of control loop performance in the design stage are listed in Table 1. In Example 1, the ranges of model parameters variation are chosen as $\eta_{K} \in[-40 \%, 40 \%] \quad, \quad \eta_{T} \in[-50 \%, 50 \%] \quad$ and $\eta_{\tau} \in[-50 \%, 50 \%]$. Here, the intervals for each parameter change is 5\%. Thus, 7497 sets of mismatched parameters are obtained and used as the outputs of BP neural networks for corresponding patterns of closedloop responses. In Example 2, the ranges are chosen as $\eta_{K} \in[-21 \%, 21 \%], \eta_{T} \in[-50 \%, 50 \%], \quad$ and $\eta_{\tau} \in[-40 \%, 40 \%], \eta_{\lambda} \in[-20 \%, 80 \%]$. Here, the interval for the change of $K$ and $\lambda$ are $3 \%$ and $10 \%$, respectively, and for the others 5\%. Thus, 58905 sets of mismatched parameters are obtained. For all examples, the closed-loop responses are obtained using Simulink. The normalized characteristic variables are calculated by the previously mentioned method and used as the inputs of BP neural networks for the corresponding patterns of closed-loop responses. For Example 1 and 2, 6 BP networks are established respectively. To evaluate the fitting effect of BP network, regression $\mathrm{R}$ values are used to measure the correlation between outputs and targets, and the closer the $\mathrm{R}$ value is to 1 , the closer the relationship between them is. $\mathrm{R}$ values for $\mathrm{BP}$ neural networks of three patterns are listed in Table 2. It is seen that all $\mathrm{R}$ values are higher than 0.97 , so the fitting effects of the BP neural networks are satisfactory.

Table 1. Characteristic variables and indices of control loop performance

\begin{tabular}{cccccccc}
\hline & \multicolumn{4}{c}{ Characteristic variables } & \multicolumn{4}{c}{ Indices of control loop performance } \\
\cline { 2 - 9 } & $C_{d 1}$ & $C_{d 2}$ & $C_{d 3}$ & $C_{d 4}$ & $I T A E_{d, s}$ & $I T A E_{d, l}$ & $M_{S d}$ \\
\hline Example 1 & 46.4 & 108.1 & 120.9 & 1.017 & 120.9 & 285.2 & 1.40 \\
\hline
\end{tabular}




\begin{tabular}{llllllll}
\hline Example 2 & 15959.3 & 27851.5 & 32657.3 & 1.016 & 32657.3 & 92749.5 & 1.63
\end{tabular}

Table 2. $\mathrm{R}$ values of BP neural network for three patterns

\begin{tabular}{cccc}
\hline & \multicolumn{3}{c}{ R values } \\
\cline { 2 - 4 } & Underdamped & Moderate-damped & Overdamped \\
\hline Example 1 & 0.999 & 0.999 & 0.998 \\
Example 2 & 0.976 & 0.999 & 0.974 \\
\hline
\end{tabular}

For each example, 100 times Monte Carlo trials are carried out to simulate the model mismatch in the monitoring stage, by randomly choosing the parameters in the following model sets, respectively:

$$
\begin{aligned}
& G_{p m}(s ; \theta)=\left\{\frac{K_{m}}{T_{m} s+1} e^{-\tau_{m} s} \mid 0.6<K<1.4,5<T<15,2.5<\tau<7.5\right\} \\
& G_{m 1}(s ; \theta)=\left\{\begin{array}{l|l}
K_{m 1} & \begin{array}{l}
1.34<K_{m 1}<2.01, \\
\left(T_{m 1} s+1\right)^{2}
\end{array} \\
16<T_{m 1}<24
\end{array}\right\}, \\
& G_{m 2}(s ; \theta)=\left\{\begin{array}{l|l}
\frac{K_{m 2}}{\left(T_{m 2} s+1\right)^{7}} & \begin{array}{l}
0.96<K_{m 2}<1.44, \\
23<T_{m 2}<32
\end{array}
\end{array}\right\}
\end{aligned}
$$

The above mismatched models obtained in the monitoring stage are estimated by the proposed method, BFGS [2] and GA method [4]. The higher order processes are estimated as SOPTD models in Example 2. The MATLAB optimization toolbox is used for the BFGS method, in which the values used for convergence are TolX $=1.0 \times 10^{-3}$, TolFun $=1.0 \times 10^{-6}$, and number of iterations $=400$.The approach to the selection of initial guesses is proposed in [3]. GA method is employed by using the routine ga in Matlab, and the option values for the algorithm are set as followed: MaxGenerations $=400$, PopulationSize $=30$, CrossoverFraction $=0.8$, MigrationFraction $=0.2$.

To assess the accuracy of estimation, the worst-case error (E) and the integral of absolute error (IAE) are selected as the measure of the estimation error in the frequency and time domain, respectively. Here, IAE is calculated from the difference between the open-loop unit step responses of actual and those of estimated process. The measure $\mathrm{E}$ is defined as:

$$
E=\max _{\omega \in\left[0, \omega_{g}\right]}\left\{\left|\frac{\hat{G}(\mathrm{j} \omega)-G(\mathrm{j} \omega)}{G(\mathrm{j} \omega)}\right| \times 100\right\}
$$

where $\hat{G}(\mathrm{j} \omega)$ and $G(\mathrm{j} \omega)$ are the frequency responses of estimated and actual process, respectively.

Figure 4-5 show the results of IAE and E values of estimated model by the mentioned three methods for Example 1-2. It is observed that all IAE and E values of the estimated model by the proposed method are at lower level in each example, along with small fluctuation among the different trials. The average values of IAE and $\mathrm{E}$ in the 100 times Monte Carlo trials are listed in Table 3. Table 3 illustrates that the average estimation error by the proposed method is lower than other two methods. The IAE values and E values of Example 2 by the proposed method are larger than those of Example 1, because the approximate error exists between the SOPTD model and higher-order process. These examples demonstrate that the proposed method provide more accurate and reliable estimation effect.

Table 3. The average estimation error measures for three methods

\begin{tabular}{ccccccc}
\hline & \multicolumn{2}{c}{ the proposed method } & \multicolumn{2}{c}{ BFGS method } & \multicolumn{2}{c}{ GA method } \\
\cline { 2 - 6 } & $I A E_{a v}$ & $E_{a v}(\%)$ & $I A E_{a v}$ & $E_{a v}(\%)$ & $I A E_{a v}$ & $E_{a v}(\%)$ \\
\hline Example 1 & 0.24 & 1.1 & 1.07 & 12.6 & 0.48 & 2.4 \\
Example 2 & 9.84 & 8.8 & 33.59 & 36 & 12 & 11 \\
\hline
\end{tabular}
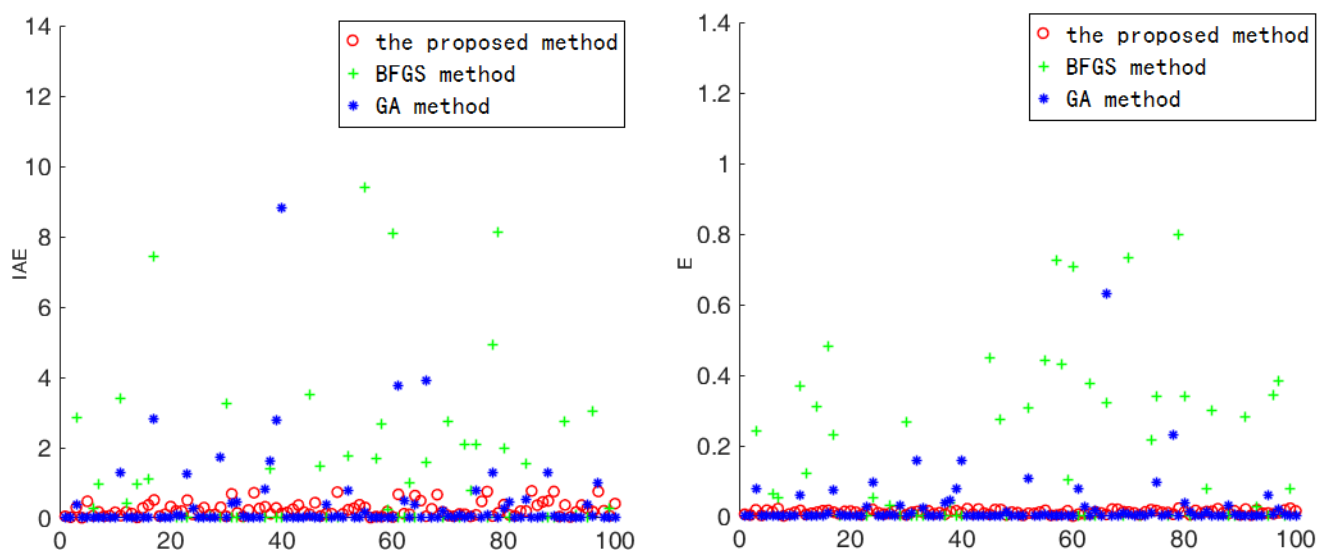

Figure 4. IAE and $\mathrm{E}$ values of estimated models in Example 1 

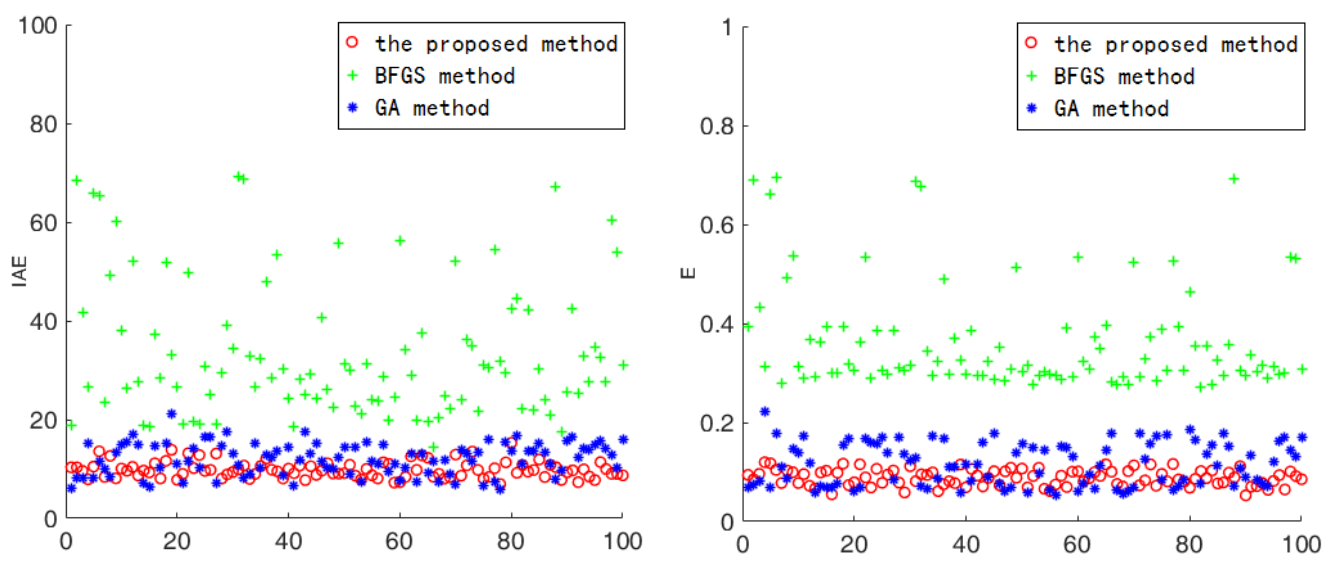

Figure 5. IAE and E values of estimated models in Example 2

For the performance monitoring, the proposed method is applied to SOPTD model in Example 2, Here, $I T A E_{d, s}, I T A E_{d, 1}$ and $M_{S d}$ listed in Table 1 are used as the performance benchmark, and $G_{p m}$ is considered as the benchmark model. Here, suppose the alarm threshold values set for the normalized indices are 3\%. For Example 2, the mismatched models of which the normalized indices are within the range of $\pm 3 \%$ are selected, and the step set-point responses and the step load disturbance responses of the corresponding control loops for these models are shown in Figure 6. Each red trajectory represents the response of a selected model. Consider two model mismatch cases in Example 2. The model mismatches and the corresponding normalized indices are listed in Table 4. The mismatch and normalized index are defined as $\eta_{\theta}=\left[\eta_{K}, \eta_{T}, \eta_{\tau}, \eta_{\lambda}\right]$ and $\eta_{I}=\left[\eta_{\text {ITAE }}, \eta_{\text {ITAE }}, \eta_{M_{S}}\right]$, respectively.

As listed in Table 4, the normalized indices in Case 1 indicate that the set-point tracking performance are deteriorated by $-1.1 \%$, and load disturbances rejection performance and loop robustness are improved by $0.04 \%$ and $2.9 \%$, respectively. As shown in Figure 6, the change of control performance in case 1 is acceptable, so that the model mismatch does not need alarm. The normalized indices in case 2 show a big degradation of both the setpoint tracking performance and load disturbances rejection performance with $76 \%$ and $51 \%$, respectively. Figure 6 shows that the changes of control performance are serious in case 2 , so that the model mismatch need to be alarmed.
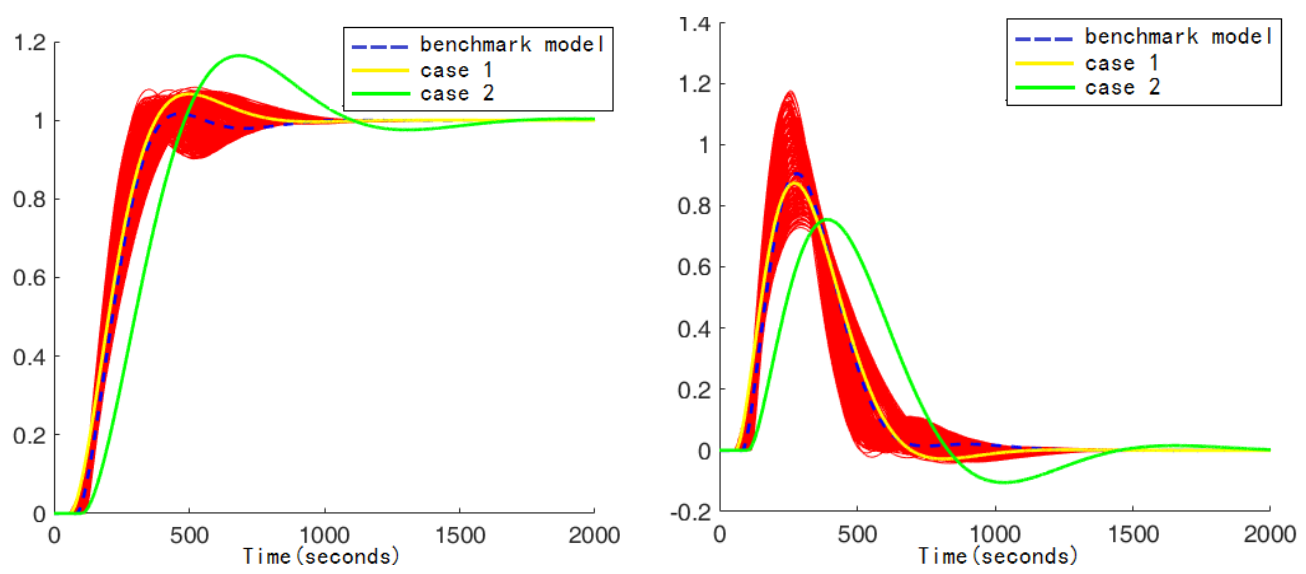

Figure 6. The closed-loop responses of the mismatched SOPTD models

Table 4. The mismatches in Case 1 and Case 2 of Example 2

\begin{tabular}{ccc}
\hline & Case 1 & Case 2 \\
\cline { 2 - 3 }$\eta_{\theta}$ & {$[18 \%, 20 \%,-30 \%, 10 \%]$} & {$[-9 \%, 45 \%, 25 \%, 70 \%]$} \\
$\eta_{I}$ & {$[-1.1 \%, 0.04 \%, 2.9 \%]$} & {$[-76 \%,-51 \%,-2.8 \%]$} \\
Status & Acceptable & Unacceptable \\
\hline
\end{tabular}




\section{Conclusion}

This study proposes a method for performance monitoring of control system in the case of MPM. First, the mismatched model is estimated by the relationship between the change of closed-loop response and model parameters built by BP neural network. Then, the indices of control performance is calculated based on the estimated model and normalized to evaluate the change of control performance. Finally, according to the acceptable variation range of the normalized indices, the serious mismatch can be alarmed. Simulation results demonstrate the efficacy in performance monitoring by the method.

\section{References}

1. Gao, X., Shang, C., Huang, D., Yang, F. (2017) A novel approach to monitoring and maintenance of industrial PID controllers. Control Engineering Practice, 64, 111-126.

2. Viswanathan, P. K., Rangaiah, G. P. (2000) Process identification from closed-loop response using optimization methods. Chemical Engineering Research \& Design, 78(4), 528-541.

3. Rajapandiyan, C., Chidambaram, M. (2012) Closedloop identification of second-order plus time delay (soptd) model of multivariable systems by optimization method. Industrial \& Engineering Chemistry Research, 51(28), 9620-9633.

4. Shin, G. W., Song, Y. J., Lee, T. B., Choi, H. K. (2007) Genetic algorithm for identification of time delay systems from step responses. International Journal of Control Automation and Systems, 5(1), 79-85.

5. Yang, Z., Seested, G. T. (2013) Time-delay system identification using genetic algorithm - part one: precise FOPDT model estimation. IFAC Proceedings Volumes, 46(20), 561-567.

6. Wang, H., Hägglund, T., Song, Z. (2012) Quantitative analysis of influences of model plant mismatch on control loop behavior. Industrial \& Engineering Chemistry Research, 51(49), 1599716006.

7. Fan, Y., Xu, Z., Chen, L. (1997) Study of adaptive fuzzy control of boiler superheated steam temperature based on dynamic mechanism analysis. Proceedings of the CSEE, 17(1), 23-28. 